\title{
Universal scaling of magnetoconductance in magnetic nanocontacts (invited)
}

\author{
S. H. Chung ${ }^{\text {a) }}$ \\ Electrical and Computer Engineering Department and Physics Department, University of Maryland, \\ College Park, Maryland 20742 and Laboratory for Physical Sciences, College Park, Maryland 20740 \\ M. Muñoz and N. García \\ Laboratorio de Física de Sistemas Pequeños y Nanotecnología, Consejo Superior de Investigaciones \\ Científicas, Serrano 144, E-28006 Madrid, Spain \\ W. F. Egelhoff \\ National Institute of Science and Technology, Gaithersburg, Maryland 20899-8552 \\ R. D. Gomez ${ }^{\text {b) }}$ \\ Electrical and Computer Engineering Department and Physics Department, University of Maryland, \\ College Park, Maryland 20742 and Laboratory for Physical Sciences, College Park, Maryland 20740
}

(Presented on 14 November 2002)

\begin{abstract}
We present results of half-metallic ferromagnets formed by atomic nanocontact of $\mathrm{CrO}_{2}-\mathrm{CrO}_{2}$ and $\mathrm{CrO}_{2}-\mathrm{Ni}$ that show as much as $400 \%$ magnetoconductance. Analysis of the magnetoconductance versus conductance data for all materials known to exhibit so-called ballistic magnetoresistance strongly suggests that the magnetoconductance of nanocontacts follows universal scaling. If the maximum magnetoconductance is normalized to unity and the conductance is scaled to the resistivity of the material, then all data points fall into a universal curve that is independent of the contact material and the transport mechanism. The analysis was applied to all available magnetoconductance data of magnetic nanocontacts in the literature, and the results agree with theory that takes into account the spin scattering within a magnetic domain wall. (c) 2003 American Institute of Physics. [DOI: 10.1063/1.1556131]
\end{abstract}

\section{INTRODUCTION}

The observation of large magnetoresistance (MR) in spin-polarized ferromagnets connected by nanoconstrictions is causing excitement in the scientific community. The effect, commonly known as "ballistic magnetoresistance" (BMR) often exceeds giant magnetoresistance (GMR) and tunneling magnetoresistance (TMR) effects at room temperature. Its application to magnetoelectronic devices such as nonvolatile memory and high performance magnetic field sensors for information storage ${ }^{1}$ is obvious, and for this reason, understanding the effect and establishing the parameters for practical device fabrication are extremely valuable.

At the time of this writing, there are several material systems in which the effect has been reported. Since 1999, García et al. have performed a series of experiments on various $3 d$ transition metals $(\mathrm{Fe}, \mathrm{Co}, \mathrm{Ni}$ ) with atomic size contacts and observed ballistic magnetoconductance (MC) $\Delta G / G$ of $280 \%,{ }^{2}$ and in electrodeposited Ni-Ni nanocontacts of $30 \mathrm{~nm}$ in size up to $700 \% \mathrm{MC}$. $^{3}$ Recently, a claim of more than $3000 \%$ was reported by Chopra and Hua using

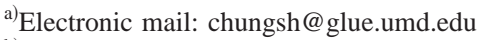

b) Author to whom correspondence should be addressed; electronic mail: rdgomez@eng.umd.edu
}

electrodeposition. ${ }^{4}$ Apart from transition metals, 500\% magnetoresistance has also been reported for magnetite $\mathrm{Fe}_{3} \mathrm{O}_{4}$ crystallites by Verluijs et al..$^{5}$ In this work, we report observations of $\mathrm{BMR}$ on half-metallic $\mathrm{CrO}_{2}$ junctions as well as on $\mathrm{CrO}_{2}-\mathrm{Ni}$ heterojunctions.

Despite differences in formulation, several theories ${ }^{6-10}$ suggest that the effect is due to spin scattering at the domain wall and is dependent of the ratio of the spin relaxation length with the domain wall width. Since the domain wall width is a function of the junction area, the effect is consequently a function of the contact area as well. At the moment, a technique with which to accurately determine the area has not yet been developed, so experimental comparison with theory is based solely on the change of

$$
\frac{\Delta G}{G}=\frac{G_{\uparrow \uparrow}-G_{\uparrow \downarrow}}{G_{\uparrow \downarrow}}
$$

or

$$
\left[\frac{\Delta R}{R}=\frac{R_{\uparrow \downarrow}-R_{\uparrow \uparrow}}{R_{\uparrow \uparrow}}\right]
$$

as a function of the conductance (or resistance). The principal ingredient in all successful experiments is the formation of very small contact areas that join two ferromagnetic elec- 
trodes. The magnetoconductance decreases rapidly with the contact area and the magnetoconductance peak occurs for nanometer size contacts. In some cases, the contact is estimated to be a few atoms wide.

In all experimental reports, apart from the common thread of observing large MR that peaks at the highest junction resistance, the resistance regimes where the maximum occurs are substantially different between different materials. In metals, the maximum occurs at one conductance quantum, $G_{0}$, (or $R_{0}=1 / G_{0}=12.9 \mathrm{k} \Omega$ ), suggesting ballistic transport through the nanoconstriction may be the dominant mechanism, whereas for oxides, the maximum is achieved at much higher resistances $(>100 \mathrm{k} \Omega$ ), well within the tunneling regime.

In this work, we will present our results on several systems, including half-metallic $\mathrm{CrO}_{2}$ and $\mathrm{CrO}_{2}-\mathrm{Ni}$ heterojunctions, as well as clarify the differences between the MR characteristics in transition metal and half-metallic oxides. We will further show that for all existing data in the literature ${ }^{2-5}$ of which we are aware the observed $\Delta G / G$ vs $G$ curves all collapse into a single curve, which has universal behavior after appropriate scaling of the conductance for the junction. We then propose that the same mechanism, i.e., spin-ballistic transport through the junction, may govern the large MR observed in different regimes of conductance in metallic $\mathrm{Ni}$, $\mathrm{Co}, \mathrm{Fe}$, and $\mathrm{CrO}_{2}$ to $\mathrm{Fe}_{3} \mathrm{O}_{4}$, which is an insulator with a Verwey transition at $118 \mathrm{~K} .{ }^{11}$ This implies that large MR in nanocontacts may be observable in a large class of material systems.

\section{EXPERIMENTAL TECHNIQUE}

A simplified schematic of the experimental setup is shown in Fig. 1. The setup is adapted from the technique given in Ref. 2. All measurements were carried out at room temperature and ambient pressure. Atomic nanojunctions are formed by putting into contact the mechanically polished ends of two nickel rods with $2 \mathrm{~mm}$ diameters. One rod is immovable whereas the other can be translated by a micrometer positioner. The contact area is varied by adjusting horizontal displacement of the movable rod. A Teflon holder is used to confine the rods in alignment, to provide mechanical rigidity, as well as to minimize thermal and magnetostriction effects. Each rod contains electromagnetic coils of several hundred turns that use $20 \mathrm{mil}$ enamel wire. One elctromagnet is driven using dc current, while the other is driven using a square wave ac current. At the tip of the nickel rods, up to 150 Oe field can be generated at $0.2 \mathrm{~A}$, which was verified using a standard Hall Gauss meter. Replacing the nickel rod with a stainless steel rod reduced the field at the tip to less than $10 \mathrm{Oe}$ at the same current conditions. To determine the conductance, a $100 \mathrm{mV}$ bias voltage was applied across the junction, and the current across the junction was converted into voltage with $10^{5}-10^{7}$ gain. We found that the junction conductance can be adjusted by translating the position of the movable rod. In the case of metal junctions, a specific value of the conductance can be maintained for several seconds, whereas for the oxide junctions, the conductance remained stable for a few minutes. At any rate, the

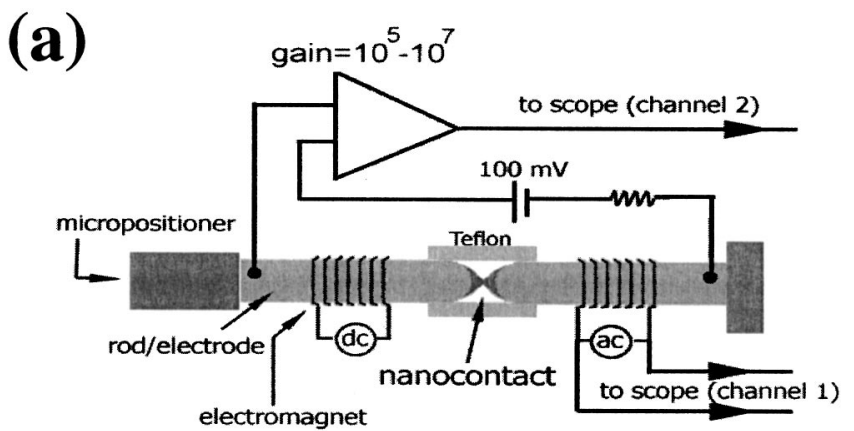

(b)

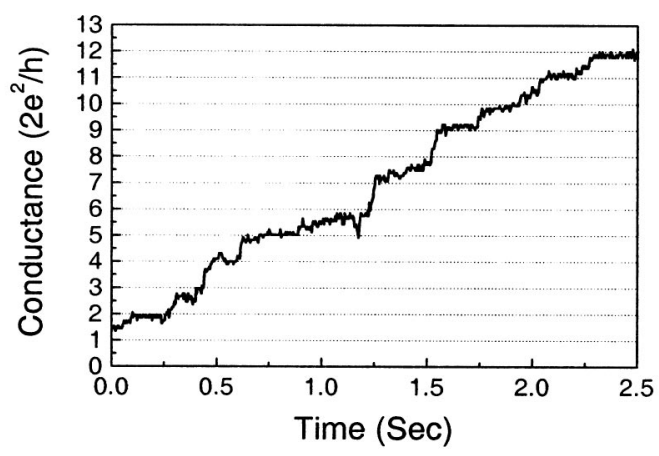

FIG. 1. (a) Schematic diagram of the experimental setup. (b) Quantized conductance of $\mathrm{Au}-\mathrm{Ni}$ nanocontacts observed with the same experimental setup.

junctions remained stable long enough to acquire magnetoconductance data. To acquire the magnetoconductance data, the junction is first stabilized to a given conductance value, with both electrodes magnetized to saturation. As soon as this is achieved, and the ac magnetic field is turned on and recorded on one digital oscilloscope channel, while the conductance is concurrently recorded on a second channel.

Using the nickel rods as support, different junction materials were investigated by depositing these substances on the tips using conventional thin film fabrication techniques for the metals, and vapor phase reaction to produce chromium dioxide $\left(\mathrm{CrO}_{2}\right)$. Since the $\mathrm{CrO}_{2}$ preparation is less known, we shall elaborate on its preparation. The samples were prepared by coating $\mathrm{CrO}_{2}(\sim 200 \mathrm{~nm})$ on $25 \mathrm{~mm}$ long $\mathrm{Ni}$ wires, using a chemical vapor deposition method in which polycrystalline $\mathrm{CrO}_{2}$ thin films were grown with a chromium trioxide $\left(\mathrm{CrO}_{3}\right)$ powder precursor. The substrate and the powder were heated under high $\mathrm{O}_{2}$ pressure, $\sim 1.4$ $\times 10^{6} \mathrm{~Pa}$, and held at $430{ }^{\circ} \mathrm{C}$ for $30 \mathrm{~min}$ before rapid quenching back to room temperature. The coercivity of the film obtained by a vibrating sample magnetometer is about 45 Oe and the resistivity by the conventional four-probe method is about $140 \mu \Omega \mathrm{cm} . \mathrm{CrO}_{2}$ is known to be half metallic, with almost complete spin polarization at the Fermi level. The complete spin polarization was predicted by a band structure calculation ${ }^{12}$ and was measured experimentally by the Andreeve reflection method. ${ }^{13}$ However, it should be noted that at room temperature the electrons can be excited over the small energy gap, which causes the breakdown of complete spin polarization. The $\mathrm{Fe}_{3} \mathrm{O}_{4}$ samples were prepared by at- 
taching small particles of magnetite crystals at the ends of the rods using conductive epoxy.

\section{EXPERIMENTAL RESULTS}

\section{A. Nanocontact formation and verification of spin-dependent transport}

To demonstrate nanocontact formation in our setup, we first performed reverse break junction experiments to show resistance quantization in normal metals. On both the magnetic and nonmagnetic samples, the resistance was continuously recorded as the junction was formed. A typical result from data of $\mathrm{Au}-\mathrm{Ni}$ nanocontact is shown in Fig. 1(b). The set of plateaus near the integer multiples of $G_{0}\left(=2 e^{2} / h\right)$ is evident, and these represent conductance quantization. ${ }^{14} \mathrm{We}$ observed this phenomenon for all normal metals $(\mathrm{Au}, \mathrm{Ni}, \mathrm{Fe})$ we investigated. The durations of those plateaus were on the order of several hundred milliseconds. Similarly, conductance fluctuations, which are common occurrences in normal metal nanocontacts, were also observed. Using the same apparatus, however, the oxide nanocontacts showed no quantization plateaus, despite the relative ease in forming stable $\mathrm{CrO}_{2}-\mathrm{CrO}_{2}$ nanocontacts with conductances much less than $G_{0}$. Resistances between 20 and $250 \mathrm{k} \Omega$ were stable for several minutes.

The behavior of the magnetoconductance in $\mathrm{CrO}_{2}$ coated electrodes is described by the raw data acquired by the digitizing oscilloscope. The data are reproduced in Fig. 2. In these traces the square current pattern corresponding to an amplitude of approximately \pm 90 Oe at the junction was applied to one of the ferromagnetic electrodes. The voltage on the $I / V$ converter and hence the current across the junction were recorded at fixed bias voltage of $100 \mathrm{mV}$. The current and hence the magnetoconductance show that provided that one of the electrodes was saturated by the dc electromagnet, the resistance of the junction followed the square wave ac magnetic field pattern of the other electrode, at the same phase and frequency. However, no change in resistance was observed when either one of the magnet sources was turned off. In Fig. 2(a), the ac alternating magnetic field was removed with the dc current on, and in Fig. 2(b), the dc bias was turned off with the ac on. Furthermore, upon removal of the field (indicated by the arrows) the conductance exhibited a rapid rise in transient followed by slowly varying relaxation to higher values.

From these results, we infer several conclusions with regard to whether the effect can be attributed solely to spindependent transport as opposed to some other classical effects. First, the loss of MR response with the removal of the dc field while maintaining the ac field is direct evidence that magnetostriction effects can be ruled out as significantly contributing to the observed changes in resistance. Otherwise, modulation of the MR corresponding to mechanical motion of the ac driven electrode would have existed. In a related experiment, we similarly found that the MR response is suppressed when a nonmagnetic barrier such as $500 \AA$ of $A u$ was formed at the junction. Second, the fact that both dc and ac fields are necessary implies that a magnetic wall or a large magnetization gradient must exist at the junction for MR to
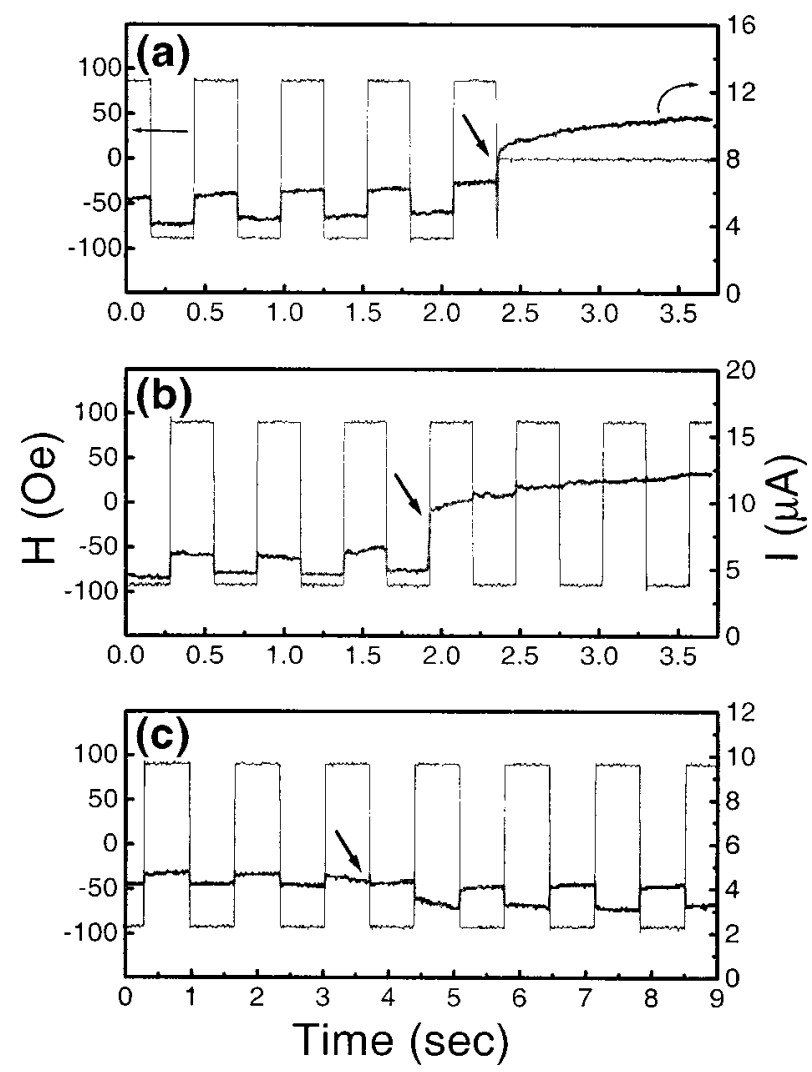

FIG. 2. Typical magnetoconductance data for $\mathrm{CrO}_{2}-\mathrm{CrO}_{2}$ nanocontacts recorded by a digitizing oscilloscope. The thin line shows the ac magnetic field applied to one of the Ni rods. The thick line shows the current through the nanocontact with a bias voltage of $100 \mathrm{mV}$. In (a) the turn-off point of the ac magnetic field is indicated by the arrow. In (b) the turn-off point of the dc magnetic field is indicated by the arrow. In (c) a high external magnetic field is applied opposite the dc magnetic field (at the arrow) and phase inversion of the magnetoconductance data occurs.

exist. With both fields on, it is easy to imagine that the $\mathrm{CrO}_{2}$ domains belonging to one electrode are pinned by the dc field, while those on the other oscillate between positive and negative saturation. By turning off one of the electromagnets, the large magnetization gradient at the contact vanishes as the magnetization of the domains at opposite sides of the junction become aligned and reoriented coherently. We further note that no MR response was observed when performing the same experiments on $\mathrm{CrO}_{2}$ films deposited on nonferromagnetic rods. The reason is that the magnitude of the field at the tip is much less and is thus insufficient to switch the magnetization of the $\mathrm{CrO}_{2}$ at the ends. In recent work by García et al., they also reported that a large external magnetic field for fixing the magnetization of electrodeposited magnetic layer on nonmagnetic electrodes is necessary for large MR response of the nanocontact. ${ }^{15}$ The third conclusion concerns the characteristic rise in transient in the conductance. One can discount the origin as due to mechanical perturbation of the system or Faraday induction since these effects would cause both an increase and decrease of conductance as well as overshoots. The fact that the transient effect is always positive suggests that, upon removal of the field, the domain wall, which initially separated the magnetization on either side of the junction is swept away, leading to lower 


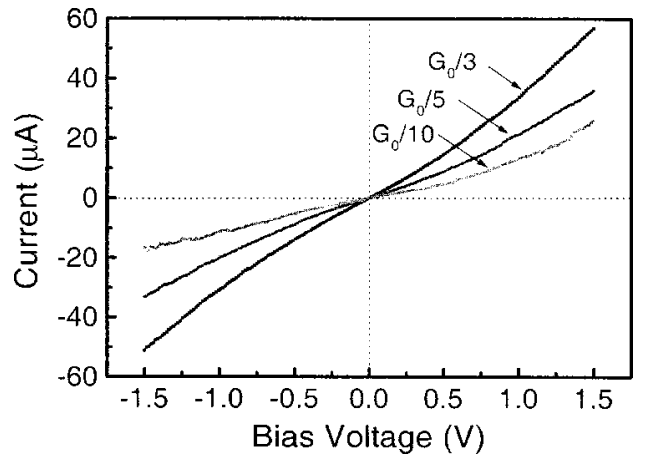

FIG. 3. Typical $I(V)$ characteristics of a $\mathrm{CrO}_{2}-\mathrm{CrO}_{2}$ nanocontact with different junction sizes.

junction resistance. The above observations are further supported by Fig. 2(c), which shows the effect of phase inversion of the MR response when a high field magnet is placed near the dc electrode. In Fig. 2(c), a strong magnet was placed in close proximity to the dc pinned electrode at about the $3 \mathrm{~s}$ mark. The phase inversion of the MR suggests that the external field from the magnet was large enough to overcome the dc pinning field of the electrode and consequently reverse the magnetization of the dc electrode. Based upon the aforementioned evidence, the MR response is thus primarily due to the spin-polarized transport between two ferromagnetic reservoirs with alternating magnetization.

We also performed $I-V$ measurements of the $\mathrm{CrO}_{2}$ junction at different junction resistances in the range of 10-150 $\mathrm{k} \Omega$ and they are shown in Fig. 3. We obtained curves similar to those in Ni-Ni (Ref. 16) and magnetite (Ref. 5) experiments, where the nonlinear behavior becomes more pronounced at higher resistance. The nonlinear behavior is not yet well understood but is nevertheless present in all materials that we studied and may be due to relatively large electron-electron interaction for small junctions. ${ }^{16}$ In the absence of pinholes in the barrier, the MR for a tunnel barrier should not depend on the size of the junction. ${ }^{16}$ Thus, the tunneling mechanism alone cannot explain the fast drop of magnetoconductance with the junction size as the data indicate.

\section{B. Comparison of $\Delta G / G$ vs $G$ results for various materials}

We reiterate that the conductance $G$ is varied by arresting the break junction at a specific contact spacing, which was accomplished by appropriate adjustment of the fine positioning micrometer. The magnetoconductance $\Delta G / G$, was defined quite simply as the difference in conductance between the parallel and antiparallel electrode magnetization orientations divided by the conductance of the antiparallel configuration.

Figure 4 presents experimental values in the form of plots of $\mathrm{MC}$ versus nanocontact conductance for $\mathrm{Ni}-\mathrm{Ni}$, $\mathrm{CrO}_{2}-\mathrm{CrO}_{2}$, and $\mathrm{CrO}_{2}-\mathrm{Ni}$ heterojunctions. We emphasize that the MC values are, by definition, identical in magnitude to the MR values. The data on Ni in Fig. 4(a) agree quite well with the results in Ref. 2. The largest MC we observed was $400 \%$ in $\mathrm{CrO}_{2}-\mathrm{CrO}_{2}$ at a conductance of $0.05 G_{0}$ [Fig.
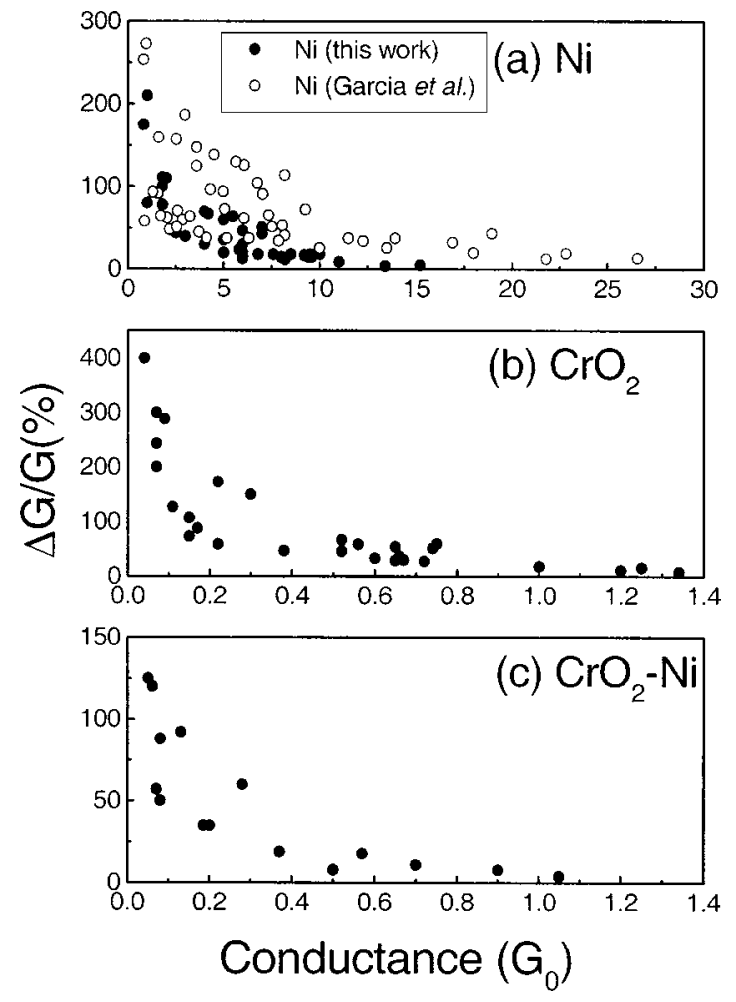

FIG. 4. Experimental data of the magnetoconductance as a function of the nanocontact conductance for (a) $\mathrm{Ni}-\mathrm{Ni}$, (b) $\mathrm{CrO}_{2}-\mathrm{CrO}_{2}$, and (c) $\mathrm{CrO}_{2}-\mathrm{Ni}$ nanocontacts.

4(b)]. By comparison, the maximum $\mathrm{MC}$ of $\mathrm{Ni}-\mathrm{Ni}$ is $210 \%$, which was obtained at $G_{0}$. The behavior of $\mathrm{CrO}_{2}-\mathrm{Ni}$ is similar to that of $\mathrm{CrO}_{2}-\mathrm{CrO}_{2}$ in that the peak $\mathrm{MC}$ occurs at very low conductance although the maximum $\mathrm{MC}$ is lower.

In anticipation of the subsequent analysis, we combine the data for all three systems by appropriate normalization of the peak conductance and scaling of the conductance with the material resistivity. A summary of the three cases is plotted in Fig. 5, which shows the normalized magnetoconductance as a function of the junction conductance. Normalization was done by setting the magnetoconductance to unity at its peak. The conductance for each data set was scaled to

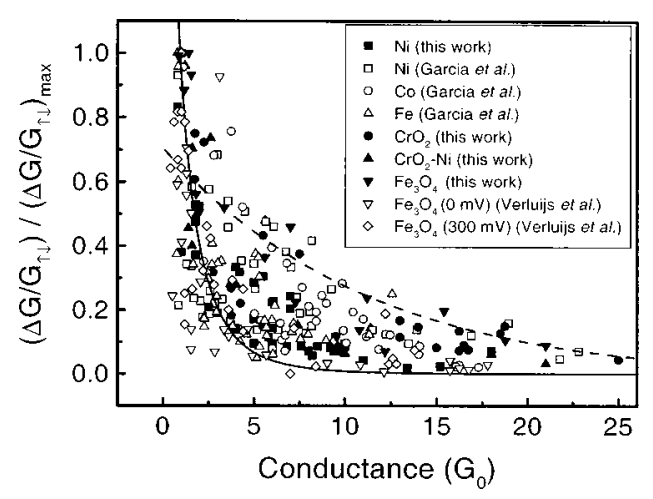

FIG. 5. Normalized magnetoconductance as a function of the nanocontact conductance scaled by the ratio of the material resitivity to the resitivity of $\mathrm{Ni}$. For both our data and that in the literature the conductances are scaled to $G_{0}$ at the peak magnetoconductance. The solid and dotted curves are when the domain wall magnetization profile varies smoothly or is constant in the domain wall, respectively (from Ref. 7). 
match that of $\mathrm{Ni}$ by multiplying the actual conductance of each nanocontact by the ratio of the resistivity of $\mathrm{Ni}$ to the resistivity of the other junction material. In the case of the $\mathrm{CrO}_{2}-\mathrm{Ni}$ heterojunction, we used the conductance of $\mathrm{CrO}_{2}$. The same aforementioned scaling procedure is applied to the conductance data of others on the metals $\mathrm{Ni}, \mathrm{Co}$ and $\mathrm{Fe},{ }^{2}$ as well as the insulator (the slope of the resistivity versus temperature is negative) $\mathrm{Fe}_{3} \mathrm{O}_{4} \cdot{ }^{5}$ Note that all the magnetoconductance curves overlap each other reasonably well. This suggests the intriguing possibility that the magnetoconductance mechanism is the same in all three different systems (transition metal, half-metallic oxide Verwey insulator) and only has to do with spin conservation in the transport process due to domain wall scattering at the constriction or contact. ${ }^{6,7}$ The normalized magnetoconductance in nanocontacts has universal behavior versus scaled conductance independent of the electron transport mechanism.

\section{ANALYSIS AND DISCUSSION}

We now analyze the data for transition metals $\mathrm{Ni}, \mathrm{Co}$, and $\mathrm{Fe}$, the half-metallic $\mathrm{CrO}_{2}$, and the Verwey insulator $\mathrm{Fe}_{3} \mathrm{O}_{4} . \mathrm{Ni}, \mathrm{Co}$, and $\mathrm{Fe}$ have similar resistivities of between 6 and $8 \mu \Omega \mathrm{cm}$ and hence scaling has little effect on the data. With $\mathrm{CrO}_{2}$, the best agreement is obtained by scaling the conductance using a scaling factor of 19 . The half-metallic $\mathrm{CrO}_{2}$ has a resistivity of $140 \mu \Omega \mathrm{cm}$ or a factor of 23 larger than that of $\mathrm{Ni}$, so the discrepancy (19 vs 23 ) is very reasonable considering that lattice disorder at a mechanically formed nanocontact could easily change the resistivity by a factor of 2 from its bulk single crystal value. The situation is less settled for the case of $\mathrm{Fe}_{3} \mathrm{O}_{4}$. For the data of Verluijs et al. ${ }^{5}$ on $\mathrm{Fe}_{3} \mathrm{O}_{4}$, the best agreement is found for a scaling factor of 30 (corresponding to bulk resistivity of 180 $\mu \Omega \mathrm{cm}$ ), and that value was used to plot our $\mathrm{Fe}_{3} \mathrm{O}_{4}$ data in Fig. 5. This compares with room temperature resistivities from 4000 to $10^{5} \mu \Omega \mathrm{cm}$, which have been reported for bulk single crystals and thin films fabricated by a variety of techniques. The reason for the apparent discrepancy is that $\mathrm{Fe}_{3} \mathrm{O}_{4}$ is complicated and the resistivity values are strongly dependent upon the crystallinity, film thickness, and fabrication conditions. Furthermore, $\mathrm{Fe}_{3} \mathrm{O}_{4}$ is an insulator below the 118 $\mathrm{K}$ Verwey transition and at higher temperatures exhibits thermally excited hopping transport of minority spin electrons between the $\mathrm{Fe}^{3+}$ and $\mathrm{Fe}^{2+}$ that coexist. Thus, the thermally activated character of the conductance means that the conductance at the nanocontact could easily change by orders of magnitude, unlike in normal metals. Therefore, it is not surprising that the scaling factor could be off by an order of magnitude.

We repeated the experiments with magnetite using the same procedures and found a maximum MR of $350 \%$ as was reported in Ref. 5. However, in contrast to those authors' results, the peak MR in our experiments occurred at a conductance value of $0.005 G_{0}$, which is about $1 / 10$ lower than previously reported. Using this, the scaling factor for our data is 200 , which implies that the resistivity at the contact is $1200 \mu \Omega \mathrm{cm}$. This is in much better agreement with the value for crystals of magnetite.
Scaling with the resistivities is the pivotal point in explaining the universality of MR in nanocontacts. According to Cabrera and Falicov theory ${ }^{6}$ of the scattering of electron spins by a domain wall (DW), discussed by Tatara and Fukuyama ${ }^{7}$ for nanocontacts, the spin transport adiabaticity increases as the DW width increases. In thick DWs the MR is reduced because the spin can rotate and align with the local magnetization as the electron travels through the wall. Conversely, for very thin domain walls $(\approx 1 \mathrm{~nm})$ the spin is almost completely conserved as the electron crosses the wall. From Refs. 6-8, the conductance for the parallel magnetization configuration, with no DW at the nanocontact, is given by the Landauer formula: ${ }^{17}$

$$
G_{\uparrow \uparrow}=\frac{2 e^{2}}{h} \sum_{i} T_{i},
$$

where $e$ and $h$ are the electron charge and Planck constant, respectively. $T_{i}$ is the transmittivity of channel $i$ at the nanocontact for spin up electrons. The conductance for the antiparallel magnetic configuration is $G_{\uparrow \downarrow}=R^{-1}$, where the resistance $R$ is

$$
R=G_{\uparrow \uparrow}^{-1}+R_{w},
$$

and the DW resistance $R_{w}$ for the nanocontact of the same metal is

$$
R_{w} \approx \frac{1}{G_{\uparrow \uparrow}} \frac{2 P^{2}}{1-P^{2}} F(P, \lambda),
$$

where $P=\left(D_{\uparrow}-D_{\downarrow} / D_{\uparrow}+D_{\downarrow}\right)$ is the polarization at the Fermi level given by the ratio of the density of states of $D_{\uparrow}$ and $D_{\downarrow}$, and $\lambda$ is the DW width.

The relative conductance is

$$
\frac{\Delta G}{G_{\uparrow \downarrow}}=\frac{G_{\uparrow \uparrow}-G_{\uparrow \downarrow}}{G_{\uparrow \downarrow}}=\frac{2 P^{2}}{1-P^{2}} F(P, \lambda),
$$

which is independent of the conductance. $F(P, \lambda)$ is the function that describes the accommodation of the spin in the DW. ${ }^{6,7}$ Note that this is unity for $\lambda \rightarrow 0$, and Eq. (4) reduces to Julliere's ${ }^{18}$ result when the spin transport is completely nonadiabatic, i.e., the spin is conserved. On the other hand, $F(P, \lambda) \rightarrow 0$ as $\lambda$ grows. This function behaves as $\exp (-\beta \lambda)$ for $\lambda \approx \lambda_{F}$ and as $1 / \alpha \lambda^{2}$ for $\lambda \gg \lambda_{F}$ where $\lambda_{F}$ is the Fermi wavelength and $\beta$ and $\alpha$ are constants. ${ }^{6,7}$ Making the reasonable assumption that $\lambda \sim d$ ( $d$ is the nanocontact cross section diameter), then $\lambda \sim N^{1 / 2}$ where $N$ is the number of conducting channels allowed in the nanocontact. The normalized result for the Ni-Ni nanocontacts is presented in Fig. 5 by the solid line for small $N$ and by the dashed line for the asymptotic value for large $N$. We note that the asymptotic limit for large $N$ uses the uniform wall limit which gives $\Delta G / G$ as being practically constant for large $G{ }^{7}$ This simple model is very appealing in light of the observation that a great majority of data points in Fig. 5 lie between these two natural limits.

It is thus clear that the behavior of the normalized MC is defined by the spin scattering function $F(P, \lambda) .{ }^{6-8}$ The value of the conductance can be obtained by Sharvin's formula: ${ }^{19}$ 


$$
G=G_{0} \frac{d^{2}}{\lambda_{F}^{2}} \gamma,
$$

where $d^{2} / \lambda_{F}^{2}$ is the number of channels and $\gamma$ the transmittivity per channel. Interpretation of the data using Eq. (5) takes into account the different material resistivities, and this is our approach to establishing general behavior of the data. The point is, as will be apparent in the next paragraph, that the MR does not need only ballisticity to have universal behavior. Diffusive, or activated transport can also have the same universal behavior because at the end of the nanocontact at which the DW scattering occurs the channel transmittivity depends on the transport process and thus on the resistivity. However, the ratio of the currents in the parallel and antiparallel configurations does not depend on how large the transmittivities are but is instead defined by the DW scattering. Therefore if $\gamma \ll 1$, then the conductance can be much smaller than $G_{0}$ even if the number of channels is larger than 1. It seems clear from our data that half-metallic oxide samples manifest this behavior whereas normal metals do not. As the $F(P, \lambda)$ is scaled by $\lambda \sim d$, then from Eq. (5) it is clear that $\lambda$ can be large while $G$ is small, and that $\gamma$ is the scaling factor that is approximately inversely proportional to the resistivity of the material.

It is important to make the distinction between "spin ballistic" and the usual notion of ballistic transport. In the former, the measure of spin ballisticity is when the spin relaxation length is longer than the domain wall width, whereas in the latter, the transport is ballistic when the scattering mean free path is larger than the contact length otherwise it is diffusive. However, we should keep in mind that the spin relaxation length can be much larger than the mean free path. ${ }^{20} \mathrm{~A}$ theoretical analysis, which makes a clear argument for large MR to occur in nanocontacts even in the diffusive regime, is published in Refs. 9 and 10. According to the quasiclassical theory of spin transport through magnetic nanocontact given by Tagirov et al., ${ }^{9,10}$ large MR can be obtained if strong spin scattering at the DW is achieved for antiparallel alignment of magnetization in the magnetic nanocontact. To realize large spin scattering, the electron spin orientation should be preserved during transit through nanocontacts. If domain wall width $\lambda$ is shorter than the mean distance between spin-flip scattering $d_{s}=v_{F} T_{s}$, where $v_{F}$ is the Fermi velocity and $T_{s}$ is the spin relaxation time, the spin can be preserved and large MR can be obtained even in the diffusive transport regime. ${ }^{9}$ Furthermore, Tagirov et al. have predicted that the nanocontact of highly spin-polarized metals such as LSMO and $\mathrm{CrO}_{2}$ would show very large MR of $1000 \%$ or higher. ${ }^{10}$ The mean free path $l$ of $\mathrm{CrO}_{2}$ is about $1.4 \mathrm{~nm}$ or less at room temperature, and this was derived from theoretical values of Lewis et al. ${ }^{21}$ The value is very small compared to that of normal metals, which is in the range of a few tens of nanometers. Therefore, diffusive transport seems to be dominant at $\mathrm{CrO}_{2}$ nanocontacts when $l$ $<d$. On the other hand, the spin orientation can be preserved even after many scattering events. ${ }^{20}$ Therefore, when the domain wall width, assumed to be of the order of the square root of the nanocontact cross section, ${ }^{22}$ is smaller than the spin-relaxation length, the electron spin relaxation cannot be accommodated by the abrupt magnetization gradient and thus strong spin scattering, i.e., high MR, can be achieved.

We note that the BMR results in Ref. 3 for electrodeposited nanocontacts belong to a different ensemble than the data described here. In those, the nanocontact contains a very thin $(<1 \mathrm{~nm})$ metallic dead magnetic layer that electrons can transit with spin conservation $(F=1)$. In that case, there is no domain wall but an abrupt change in magnetization on opposite sides of the dead layer occurs. Since no domain wall of variable width exists at the nanocontact the results scale entirely differently. ${ }^{17}$

\section{CONCLUSIONS}

We have shown that all existing normalized magnetoconductance versus conductance data that we know of on mechanically formed nanocontacts behave the same for when the conductance is scaled according to the resistivity of the material. The behavior is dominated by spin scattering at the domain wall and is controlled by the domain wall thickness. The conductance is proportional to the area of the nanocontact times the transmittivity of the nanocontact. The transmittivity of the nanocontact is approximately inversely proportional to the bulk resistivity of the material.

\section{ACKNOWLEDGMENTS}

This work was supported by NSF Grant Nos. ECS 0115327 and ECS9984797 CAREER Award, and by the Spanish DGICyT. Support by the University of Maryland, College Park, NSF, and MRSEC is also gratefully acknowledged.

${ }^{1}$ G. A. Prinz, Science 282, 1660 (1998); E. Y. Tsymbal and D. G. Pettifor, Solid State Phys. 56, 113 (2001).

${ }^{2}$ N. García, M. Muñoz, and Y.-W. Zhao, Phys. Rev. Lett. 82, 2923 (1999); Appl. Phys. Lett. 76, 2586 (2000); Y.-W. Zhao, M. Muñoz, G. Tatara, and N. García, J. Magn. Magn. Mater. 223, 169 (2001).

${ }^{3}$ N. García et al., Appl. Phys. Lett. 79, 4550 (2001).

${ }^{4}$ H. D. Chopra and S. Z. Hua, Phys. Rev. B 66, R020403 (2002).

${ }^{5}$ J. J. Verluijs et al., Phys. Rev. Lett. 87, 026601 (2001).

${ }^{6}$ G. G. Cabrera and L. M. Falicov, Phys. Status Solidi B 61, 539 (1974).

${ }^{7}$ G. Tatara and H. Fukuyama, Phys. Rev. Lett. 72, 772 (1994); G. Tatara, Y.-W. Zhao, M. Muñoz, and N. García, ibid. 83, 2030 (1999).

${ }^{8}$ J. B. A. N. H. van Hoof et al., Phys. Rev. B 59, 138 (1999).

${ }^{9}$ L. R. Tagirov, B. P. Vodopyanov, and K. B. Efetov, Phys. Rev. B 63, 104428 (2001).

${ }^{10}$ L. R. Tagirov et al., Phys. Rev. B 65, 214419 (2002).

${ }^{11}$ S. Chikazumi, Physics of Ferromagnetism, 2nd ed. (Clarendon-Oxford Science, Oxford, 1997), p. 205.

${ }^{12}$ K. Schwarz, J. Phys. F: Met. Phys. 16, L211 (1986); R. A. de Groot and F. M. Mueller, Phys. Rev. Lett. 50, 2024 (1983); M. A. Korotin, V. I. Anisimov, D. I. Khomskii, and G. A. Sawatzky, ibid. 80, 4305 (1998).

${ }^{13}$ R. J. Soulen, Jr. et al., Science 282, 85 (1998).

${ }^{14}$ J. I. Pascual et al., Phys. Rev. Lett. 71, 1852 (1993).

${ }^{15}$ N. García et al., Appl. Phys. Lett. 80, 1785 (2002).

${ }^{16}$ N. García, Appl. Phys. Lett. 77, 1351 (2000); G. G. Cabrera and N. García, ibid. 80, 1782 (2002).

${ }^{17}$ R. Landauer, Philos. Mag. 21, 863 (1970).

${ }^{18}$ M. Julliere, Phys. Lett. 54A, 225 (1975).

${ }^{19}$ Yu. V. Sharvin, Zh. Eksp. Teor. Fiz. 48, 984 (1965) [ Sov. Phys. JETP 21, 655 (1965)].

${ }^{20}$ M. Johnson and R. H. Silsbee, Phys. Rev. Lett. 55, 1790 (1985); G. A. Prinz, Phys. Today 48, 58 (1995).

${ }^{21}$ S. P. Lewis, P. B. Allen, and T. Sasaki, Phys. Rev. B 55, 10253 (1997).

${ }^{22}$ P. Bruno, Phys. Rev. Lett. 83, 2425 (1999). 
Journal of Applied Physics is copyrighted by the American Institute of Physics (AIP). Redistribution of journal material is subject to the AIP online journal license and/or AIP copyright. For more information, see http://ojps.aip.org/japo/japcr/jsp

Copyright of Journal of Applied Physics is the property of American Institute of Physics and its content may not be copied or emailed to multiple sites or posted to a listserv without the copyright holder's express written permission. However, users may print, download, or email articles for individual use. 